\title{
Genomic investigation reveals contaminated detergent as the source of an ESBL-producing Klebsiella michiganensis outbreak in a neonatal unit
}

\author{
Authors: \\ Paul Chapman ${ }^{1,2,3 \#}$, Brian M. Forde ${ }^{4 \#}$, Leah W. Roberts ${ }^{4}$, Haakon Bergh ${ }^{5}$, Debra \\ Vesey $^{2}$, Amy V. Jennison ${ }^{6}$, Susan Moss ${ }^{6,7}$, David L. Paterson ${ }^{1,7}$ Scott A. Beatson ${ }^{4}$, \\ Patrick N. A. Harris ${ }^{5,7^{\star}}$
}

\section{Affiliations:}

1. Infectious Diseases Unit, Royal Brisbane and Women's Hospital, Herston, QLD, Australia

2. Infection Prevention and Control, Caboolture Hospital, QLD, Australia

3. QIMR Berghofer, Herston, QLD, Australia

4. School of Chemistry and Molecular Biosciences, The University of Queensland, Brisbane, QLD, Australia

5. Microbiology Department, Central Laboratory, Pathology Queensland, Royal Brisbane \& Women's Hospital, QLD, Australia

6. Public Health Microbiology, Forensic and Scientific Services, Queensland Health, Brisbane, QLD, Australia

7. University of Queensland, Faculty of Medicine, UQ Centre for Clinical Research, Royal Brisbane \& Women's Hospital, QLD, Australia

\author{
\# Paul Chapman and Brian M. Forde contributed equally to this work \\ *Corresponding author: \\ Dr. Patrick N. A. Harris \\ University of Queensland Centre for Clinical Research
}


Building 71/918 Royal Brisbane \& Women's Hospital Campus, Herston, QLD, 4029

Email:p.harris@uq.edu.au

Tel: $+61(0) 733466081$

\section{Alternative corresponding author:}

A/Prof. Scott A. Beatson

School of Chemistry and Molecular Biosciences

Building 76 Cooper road, The University of Queensland, St Lucia, QLD 4072

Email: s.beatson@uq.edu.au

Tel: +61 (0) 733654863

Running title: ESBL-K. michiganensis neonatal outbreak

Word count: 3,051

Key words: Klebsiella oxytoca, Klebsiella michiganensis, whole genome sequencing, extended-spectrum $\beta$-lactamase, outbreak 
bioRxiv preprint doi: https://doi.org/10.1101/568154; this version posted November 27, 2019. The copyright holder for this preprint (which was not certified by peer review) is the author/funder, who has granted bioRxiv a license to display the preprint in perpetuity. It is made available under aCC-BY-NC-ND 4.0 International license.

\section{Key points:}

- K. michiganensis can be misidentified as K. oxytoca and is probably underrecognized as a nosocomial pathogen

- Whole genome sequencing of neonatal and environmental isolates during an outbreak of ESBL-producing $K$. michiganensis confirmed contaminated detergent and sinks to be the source 


\section{Abstract}

Background: Klebsiella species are problematic pathogens in neonatal units and may cause outbreaks, for which sources of transmission can be challenging to elucidate. We describe the use of whole genome sequencing (WGS) to investigate environmental sources of transmission during an outbreak of extended-spectrum- $\beta$ lactamase (ESBL)-producing Klebsiella michiganensis colonizing neonates.

Methods: Ceftriaxone-resistant Klebsiella spp. isolated from neonates (or their mothers) and the hospital environment were included. Short-read (Illumina) and longread (MinION, Oxford Nanopore Technologies) sequencing was used to confirm species taxonomy, define antimicrobial resistance genes and determine phylogenetic relationships using single nucleotide polymorphism (SNP) profiling.

Results: A total of 21 organisms (10 patient-derived and 11 environmental isolates) were sequenced. Standard laboratory methods identified the outbreak strain as an ESBL-producing Klebsiella oxytoca, but taxonomic assignment from WGS data suggested closer identity to Klebsiella michiganensis. Strains isolated from baby bath drains and multiple detergent dispensing bottles were either identical or closely related by SNP comparison. Detergent bottles contaminated by $K$. michiganensis had been used for washing milk-expressing equipment. No new cases were identified once the detergent bottles were removed and the baby baths decommissioned.

Conclusions: Environmental reservoirs may be an important source in outbreaks of multi-drug resistant organisms. WGS, in conjunction with traditional epidemiological investigation, can be instrumental in revealing routes of transmission and guiding infection control responses.

(214 words) 


\section{Introduction}

Outbreaks of ESBL-producing Enterobacterales within neonatal intensive care units are most commonly caused by Klebsiella species and may be associated with significant morbidity and mortality (1). Klebsiella michiganensis was first identified from a toothbrush holder in a home in Michigan and was initially identified as Klebsiella oxytoca, to which it is closely related (99\% nucleotide sequence identity in the 16S rRNA gene sequence).(2) Since characterization, $K$. michiganensis has been reported in clinical settings, including as a cause of diarrhea in a hematopoietic stem cell transplant recipient (3), from a sample of fluid from an abdominal fistula (4), in an investigation of hospital-acquired colonization by a New Delhi metallo- $\beta$ lactamase-producing organism (5) and as an invasive, Klebsiella pneumoniae carbapenemase (KPC)-producing pathogen causing bloodstream infection(6). While the role of $K$. oxytoca in nosocomial outbreaks is established (7-11), there have been no reported outbreaks involving $K$. michiganensis to date.

Similar to K. oxytoca, K. michiganensis carries a chromosomally-encoded OXY-type (Ambler class A) $\beta$-lactamase $(12,13)$ (also labelled as $\mathrm{K} 1$ in $K$. oxytoca (14)) which mediates resistance to amino- and carboxy-penicillins (e.g. ampicillin, ticarcillin, temocillin). Over-expression of OXY enzymes arising from mutations in regulatory genes (15-17) can lead to phenotypic resistance to multiple $\beta$-lactams. Constitutive hyperproducers may also be selected during antibiotic therapy.(18) OXY-type enzymes may give a false-positive result on extended-spectrum $\beta$-lactamase (ESBL) confirmation testing by the clavulanate combination disk method (19), yet are not 'classical' ESBLs (i.e. Bush-Jacoby Group 2be enzymes (20)). 
Outbreaks of multi-drug resistant (MDR) organisms in neonatal units are of significant concern as empirical antibiotic strategies defined in most guidelines may have limited efficacy against MDR gram-negative bacilli. Hospitalized newborns, especially when premature, are at risk for infection caused by Klebsiella spp.(21), which account for a significant proportion of nosocomially-acquired neonatal infections, particularly in developing countries.(22)

We describe an outbreak of ESBL-producing K. michiganensis (initially misidentified as $K$. oxytoca) occurring within a neonatal unit, declared after three apparently unrelated colonized cases were identified. The admissions did not overlap in time and preliminary examination failed to reveal any likely cross contamination events. We explored the utility of whole genome sequencing (WGS) in conjunction with a standard outbreak investigation to help resolve the source of the outbreak and guide interventions to successfully halt transmission.

\section{Material and methods}

\section{Setting}

Caboolture Base Hospital $(\mathrm{CBH})$ is a 280 bed regional hospital in South East Queensland, Australia. The Special Care Nursery (SCN) provides care for neonates greater than 32 weeks gestation or a birth weight of $\geq 1500 \mathrm{~g}$. The unit was designed to accommodate 12 neonates. However, due to demand is regularly forced to operate up to 14 beds. Active surveillance (rectal swabs) for multi-resistant organisms (MROs) including methicillin resistant Staphylococcus aureus (MRSA), multi-resistant Gram-negative (MRGN) bacteria and vancomycin resistant Enterococcus (VRE) is performed weekly and on all transfers in to the unit. All cases of MRO colonization or infection are reviewed by the infection control (IC) team and 
contact isolation is employed for all MRSA, VRE and MRGN (except ESBL-producing Escherichia coli).

\section{Definitions, data collection and infection control responses}

After declaration of the outbreak, enhanced surveillance was introduced with rectal swabbing of all SCN patients every 48 hours. All neonates with positive screening swabs were isolated in single rooms (when available) and with contact precautions (as per routine policy). Mothers of colonized babies were also screened. Direct observation of IC procedures and hand hygiene performance was performed by independent Hand Hygiene Australia (HHA) accredited IC staff. SCN procedures and policy compliance were reviewed.

\section{Microbial Surveillance}

Rectal swabs were collected and plated onto selective chromogenic media (chromID ${ }^{\mathrm{TM}}$ ESBL agar; bioMérieux) and examined after 18-24 hours incubation in $\mathrm{O}_{2}$. Colonies which grew on selective media were identified by matrix-associated laser desorption/ionization-time-of-flight mass spectrometry (MALDI-TOF) (Vitek MS, bioMérieux) and antibiotic susceptibility determined using the Vitek2 system (bioMérieux). ESBL production was confirmed using combination disk diffusion, where an increase in the zone of inhibition $\geq 5 \mathrm{~mm}$ by the addition of clavulanic acid $(10 \mu \mathrm{g})$ to Cefotaxime $(30 \mu \mathrm{g})$ or Ceftazidime $(30 \mu \mathrm{g})$ disks suggests ESBL production.(23) After the incident case (case 1) was identified, any ceftriaxoneresistant $K$. oxytoca from neonatal cultures of any body-site were stored, and following the declaration of an outbreak (case 3) all stored and prospective isolates were sent for WGS. For comparison, additional isolates were sequenced, including ESBL-producing $E$. coli and ceftriaxone-susceptible $K$. oxytoca from neonates, as well as any $K$. oxytoca identified from maternal samples. An outbreak case was 
defined as any individual with $K$. oxytoca cultured from any site, which was determined to be resistant to ceftriaxone by Vitek2 (irrespective of ESBL phenotypic confirmatory testing).

\section{Environmental screening}

Swabs were collected from sinks, drains, aerators of faucets, soap dispensers, damp surfaces, high use equipment and the humidification apparatus of humidicribs; and samples of consumables such as shampoo, paraffin and ultrasound gel were collected and plated onto selective chromogenic media and processed in the same way as the patient screening samples (see Microbial surveillance methods).

\section{Whole Genome Sequencing}

All $K$. oxytoca isolates, from neonates, their mothers or the environment were submitted for WGS at the Queensland Health Forensic Scientific Services (FSS) laboratory in 5 batches between January $29^{\text {th }} 2018$ and March $23^{\text {rd }} 2018$. Genomic DNA was extracted using QIAamp DNA mini kits (Qiagen, Australia) and quantified by spectrophotometry (NanoDrop; ThermoFisher) and fluorometry (Quant-iT; ThermoFisher). Paired-end DNA libraries were prepared using Nextera XT kits (Illumina; Australia) and WGS was performed using Illumina NextSeq (150 bp paired end).

Using Nanopore sequencing (MinION; Oxford Nanopore Technologies) we assembled the complete genome of strain M82255 for reference. In brief, $1.5 \mu \mathrm{g}$ of DNA was used as input for the 1D sequencing by ligation kit (SQK-LSK108) as per manufacturer's instructions. The final library was loaded onto a FLO-MIN106 R9.4.1 flow cell and run for approximately 40 hours on a MinION. 
In silico multilocus sequencing typing, single nucleotide polymorphism (SNP) typing and resistance gene detection

Sample analysis was undertaken using the custom Queensland Genomics Health Alliance (QGHA) infectious diseases genomic analysis pipeline (version: dev-0.4.0). In brief, raw Illumina sequencing data was quality trimmed using Trimmomatic (version 0.36).(24) In silico sequence typing (ST) was preformed using SRST2 and the K. oxytoca typing scheme available from PubMLST (https://pubmlst.org/). Single nucleotide polymorphism (SNP) profiling and determination of core genome SNPS was undertaken by aligning the trimmed Illumina sequencing reads for each sample to the complete genome of M82255 using Nesoni (version 0.132) (https://github.com/Victorian-Bioinformatics-Consortium/nesoni). Resistance gene profiling was performed by screening the trimmed sequence reads for each isolate against the ARG-ANNOT resistance gene database using SRST2 $(25,26)$. Only genes with a minimum of $90 \%$ nucleotide sequence identity and $90 \%$ sequence coverage were reported.

\section{Phylogenetic analysis}

A list of polymorphic positions conserved in all strains was established using Nesoni nway. Polymorphic substitution sites were concatenated to produce an alignment that was used to reconstruct the phylogeny. A recombination filtered, Maximum Likelihood phylogenetic tree was estimated using Gubbins version 2.2.0 (http://sanger-pathogens.github.io/gubbins/)(27) for the SNP alignments under the generalized time reversible (GTR) nucleotide substitution model with gamma correction for among site rate variation (ASRV).

\section{Accession numbers}


Genome data has been deposited to NCBI under Bioproject PRJNA512395. Raw Nanopore and Illumina sequence read data has been deposited to the Sequence Read Archive (accessions: SRR89420303-SRR8420330). The complete genome of M82255 has been deposited to GenBank (accessions: CP035214-CP035216)

\section{Ethics}

WGS activities for pathogen surveillance and infection control are covered by the Queensland Genomics Health Alliance (QGHA) clinical demonstration project (HREC/17/QFSS/6), with approval for waiver of consent.

\section{$\underline{\text { Results }}$}

\section{Species identification}

Initial species characterization, using MALDI-TOF, identified strain M82255 as Klebsiella oxytoca. Taxonomic assignment was confirmed in silico using Kraken (28) by comparison of sequence read data for M82255 against the NCBI RefSeq database containing $\sim 25,000$ complete bacterial, archaeal and viral genomes. However, after submission of the complete genome of M82255 to GenBank, a quality control test of the taxonomic assignment using average nucleotide identity (ANI) (29) revealed the genome of $\mathrm{M} 82255$ to be $98.903 \%$ identical to K. michiganensis $(91.1 \%$ genome coverage) but only $92.455 \%$ similar to $K$. oxytoca. Further biochemical tests were performed on M82255 to identify its position within the genus Klebsiella (Supplementary table S1). Similar to the expected biochemical profile of $K$. michiganensis, M82255 was unable to produce urease (2) confirming the results of the ANI approach. Consequently, M82255 was reclassified as K. michiganensis.

Incident cases 
From the incident case to identification of the final case 188 days later, there were ten neonates colonized with $K$. michiganensis (Figure 1). One neonate was simultaneously co-colonized with ESBL-producing E. coli. During this period there were approximately 880 births and 163 admissions to the special care nursery $(\mathrm{SCN})$. There were no cases of clinical infection caused by $K$. michiganensis. The average length of stay in SCN was 15.7 (range 5-35) days and there was a mean of 7.9 (range 2-15) days from admission to SCN to acquisition of ESBL-K. michiganensis. One patient neonatal sample was obtained from another hospital facility following discharge.

\section{Infection Control Observation}

Direct observation of the SCN infection control (IC) procedures revealed that all staff hand hygiene $(\mathrm{HH})$ performance was above the national benchmark $(80 \%)$. However, significant opportunities for cross contamination (breakdown in $\mathrm{HH}$, leaning on equipment, failure to observe contact isolation procedures) were noted in relation to non-staff (families, friends and SCN occupants). Additionally, incomplete compliance with microbial surveillance cultures on admission was noted the start of the outbreak.

\section{Environmental Screening}

Exhaustive screening of the SCN unit on multiple occasions failed to detect a source of environmental contamination. Environmental sampling was then extended to the maternity ward and birth suite. ESBL-producing K. michiganensis was identified from a baby-bath drain in a multi-purpose utility room containing laundry equipment (washing machine and laundry dryer), and a pair of baby baths (no longer used for bathing). However, an epidemiological link between the multi-purpose room in maternity and the colonized cases in SCN was not evident. 
During IC unit observation it was noted that the hospital's volunteer flower service used the multi-purpose room for cleaning flower vases. The flower services equipment was then sampled and a second $K$. michiganensis environmental isolate was retrieved from a sample of the flower service's dishwashing detergent. The hospital's detergent (Cleantec Emmy; Ecolab, Australia) was bought as bulk concentrate and then decanted into reusable detergent bottles for use around the hospital. Each drum of concentrate lasted approximately one month. $K$. michiganensis was not identified in the bulk concentrate at the time of testing, however sampling of detergent from around the hospital identified $K$. michiganensis from 7 of 13 bottles.

\section{Whole genome sequencing}

A total of 21 organisms (10 patient-derived isolates, from 10 individuals and 11 environmental isolates) were included in the genomic analysis (Table 1). A single non-ESBL producing $K$. oxytoca (M8843) was identified from a maternal rectal swab and found to belong to the ST50 lineage and was excluded from further study and not considered part of the outbreak. All K. michiganensis strains possessed chromosomal OXY-type $\beta$-lactamases, as well as SHV-2 ESBL (in addition to LEN and TEM-1B $\beta$-lactamases). Resistance genes for aminoglycosides (aac3-Ild, aph(3')-la, strA/B) and sulphonamides (sul1) were also detected.

To investigate the relationship between outbreak isolates at single nucleotide level reads from each isolate were mapped to the complete genome of $K$. michiganensis M82255. Phylogenetically, the K. michiganensis isolates formed two distinct clusters separated by 15 core genome SNPs (Figure 1; Supplementary table S2). Cluster 1 (SCN-C1 from this point on) was composed exclusively of environmental isolates $(n=10)$. Cluster $2(\mathrm{SCN}-\mathrm{C} 2)$ was composed of the 10 patient isolates and a single environmental isolate, M82256. 
WGS and an in-depth epidemiological investigation were used to determine if $K$. michiganensis transmission resulted from direct patient-to-patient transfer or through contact with a common environmental reservoir.

Between Jan $6^{\text {th }}$ and March $14^{\text {th }}$ (when the outbreak resolved) 6 colonized patients had overlapping stays in the SCN (Figure 1), four of whom were resident at the same time providing opportunity for isolate transmission between neonates. However, when WGS was used to establish relationships between these isolates there was no genetic evidence to support patient-to-patient transmission. SCN-C2 is composed of a tight cluster of closely related isolates (mean pairwise SNP distance of 3.3 SNPs).

Central to this cluster is a group of 3 isolates indistinguishable at the core genome SNP level. The cluster was composed of two isolates (M8431 and M82629) isolated from case 2 and case 10 respectively (hospital admissions separated by 147 days), and a single environmental isolate (M82256) collected from a detergent located in the SCN milk room. The remaining 8 patient isolates diverge directly from this central group but possess no common discriminatory SNPs, suggesting that each colonized patient independently acquired $K$. michiganensis from a common source, in all probability the SCN milk room detergent.

Within SCN-C1, environmental isolates collected from a baby bath drain (M8848) and detergent samples from 4 of the 8 tested detergent dispensing bottles (M82257, M82624, M82627 and M82628) were indistinguishable at the core genome level. The relatedness of isolates collected from the detergent suggests that contamination from a central source was likely responsible for introducing $K$. michiganensis into the hospital, possibly during dispensing of the bulk detergent or from the drum itself. Testing of the bulk detergent drum did not identify any biological contaminants. However, the high number of SNPs (14 core genome SNPs) separating 
environmental and patient isolates suggests that the outbreak strain may have been present in the local environment for some time.

\section{Outbreak Management}

All identified cases were isolated under contact precautions. After isolation of $K$. michiganensis in the multi-purpose room, it was immediately decommissioned and refurbished as a single use facility. Redundant equipment (baby-baths, laundry equipment) was removed and the flower service was temporarily ceased. Reusable detergent bottles from across the hospital were destroyed and the detergent supply switched to pre-filled single use detergent bottles.

As the contamination of the detergent bottles may have originated from contaminated concentrate, a search for similar cases of ESBL-producing $K$. michiganensis (or $K$. oxytoca) at other hospitals served by Pathology Queensland was performed using the statewide laboratory information system, however there was no indication of unrecognized outbreaks at other facilities.

\section{Discussion}

Klebsiella species are frequently implicated in outbreaks in the neonatal intensive care setting, although sources are not always evident despite extensive investigation and screening (30). For example, outbreaks of multi-drug resistant $K$. oxytoca have been previously linked to environmental reservoirs, with sources described as varied as handwashing sinks (7), wastewater drainage systems (9), water humidifiers for neonatal incubators (16), transducers used for blood pressure monitoring (31), and washing machines.(11) Contaminated medical solutions such as heparin (32), sodium chloride (33) or insulin (8) have also been described as a source. 
Nosocomial outbreaks of $K$. michiganensis have not been reported previously. However, based on the average nucleotide identity (ANI) approach (34), numerous strains previously identified as $K$. oxytoca have now been reclassified $K$. michiganensis, including a carbapenem resistant strain, E718 (GenBank accession: CP003683), isolated from the abdomen of a renal transplant patient (35). In fact, of the seven complete K. michiganensis genomes on GenBank, including M82255 from the outbreak described here, all were originally classified as $K$. oxytoca. Consequently, it is possible that hospital outbreaks of $K$. michiganensis may go undetected as they are prone to misclassification.

Contaminated disinfectant has previously been recognized as a potential source of $K$. oxytoca sepsis in hospitalized infants.(36) Organism factors such as a mucoid phenotype via enhanced capsule formation may enable the bacteria to survive in this environment.(36) Additionally, it has been suggested that in Klebsiella, outermembrane proteins, such as peptidoglycan-associated lipoprotein (Pal) and murein lipoprotein (LppA), contribute to resistance against detergents through modulating the integrity and selective impermeability of the cell membrane independently of LPS and capsule.(37) Strains of $K$. oxytoca have been described which are able to withstand active ingredients of commonly used detergents, such as sodium dodecyl sulfate (SDS).(38)

This report clearly demonstrates the effectiveness of high-throughput WGS as a tool to enhance and support established infection control outbreak responses. The high resolution available with WGS to compare outbreak strains was sufficient to rule out patient-to-patient transmission and suggested that an environmental reservoir might be the source of infection. The ensuing IC investigation successfully identified several environmental sites contaminated with $K$. michiganensis. WGS of the environmental isolates linked them, unequivocally, to patient isolates and identified a 
liquid detergent bottle in the milk dispensing room as the most likely source for onward transmission in the SCN. Washing detergent in the milk room was used by mothers to clean milk-expressing equipment resulting in cross-contamination between this equipment and expressed milk, which resulted in neonate colonization. Although the initial source of the contamination could not be confirmed, $K$. michiganensis isolates related to the outbreak were identified in liquid detergent dispensers located at different sites throughout the hospital. Liquid detergent dispensers are refilled from a central bulk drum of detergent concentrate. However, at the time of the outbreak the bulk concentrate was negative for $K$. michiganensis. It is possible that either a contaminated drum was introduced into the hospital, its contents dispensed and the drum disposed of prior to the outbreak or contamination occurred.

The hospital procedures and practice for preparation of cleaning materials were reviewed. It became apparent that detergent bottles would often be "topped-up" when approaching empty, rather than being emptied, washed out and left to dry before refilling. This practice would have allowed propagation of the organism in the detergent bottles after contamination. In response to this re-usable detergent bottles around the hospital were collected and destroyed and single use pre-diluted detergent bottled were sourced. Although the topping up of detergent bottles was non-compliant with existing procedures, from the point of view of the maintenance staff is was intuitive and convenient. This gap between "practice imagined" and "practice performed" illustrates the importance of direct observation of staff practice and procedures when investigating an outbreak, the importance of all hospital staff to understand the infection control policies relevant to them and for a well-resourced, active hospital infection control team.

\section{Conclusions}


Environmental reservoirs are increasingly recognized as a source of outbreaks involving MDR pathogens. We demonstrate how whole genome sequencing, in conjunction with standard epidemiological investigation, was instrumental in elucidating the source of ESBL-producing $K$. michiganensis in a neonatal unit and directing effective measures to interrupt transmission. Liquid detergent can become contaminated with pathogenic bacteria and should be considered as a potential source in such outbreaks.

\section{Acknowledgements}

We thank the scientists at Pathology Queensland for their assistance in the laboratory work.

\section{Financial support}

This work was supported by funding from the Queensland Genomics Health Alliance (now Queensland Genomics), Queensland Health, Queensland Government. L.W.R was supported by an Australian Government Research Training Program (RTP) Scholarship. P.N.A.H was supported by a National Health and Medical Research Council (NHMRC) Early Career Fellowship (GNT1157530).

Potential conflicts of interest. P.N.A.H has received research grants from MSD and Shionogi Ltd, outside of the submitted work, and speaker's fees from Pfizer paid to the University of Queensland. D.L.P reports receiving grants and personal fees from Shionogi and Merck Sharp and Dohme and personal fees from Pfizer, Achaogen, AstraZeneca, Leo Pharmaceuticals, Bayer, GlaxoSmithKline, Cubist, Venatorx, and Accelerate. The other authors have no conflicts of interest to declare. 


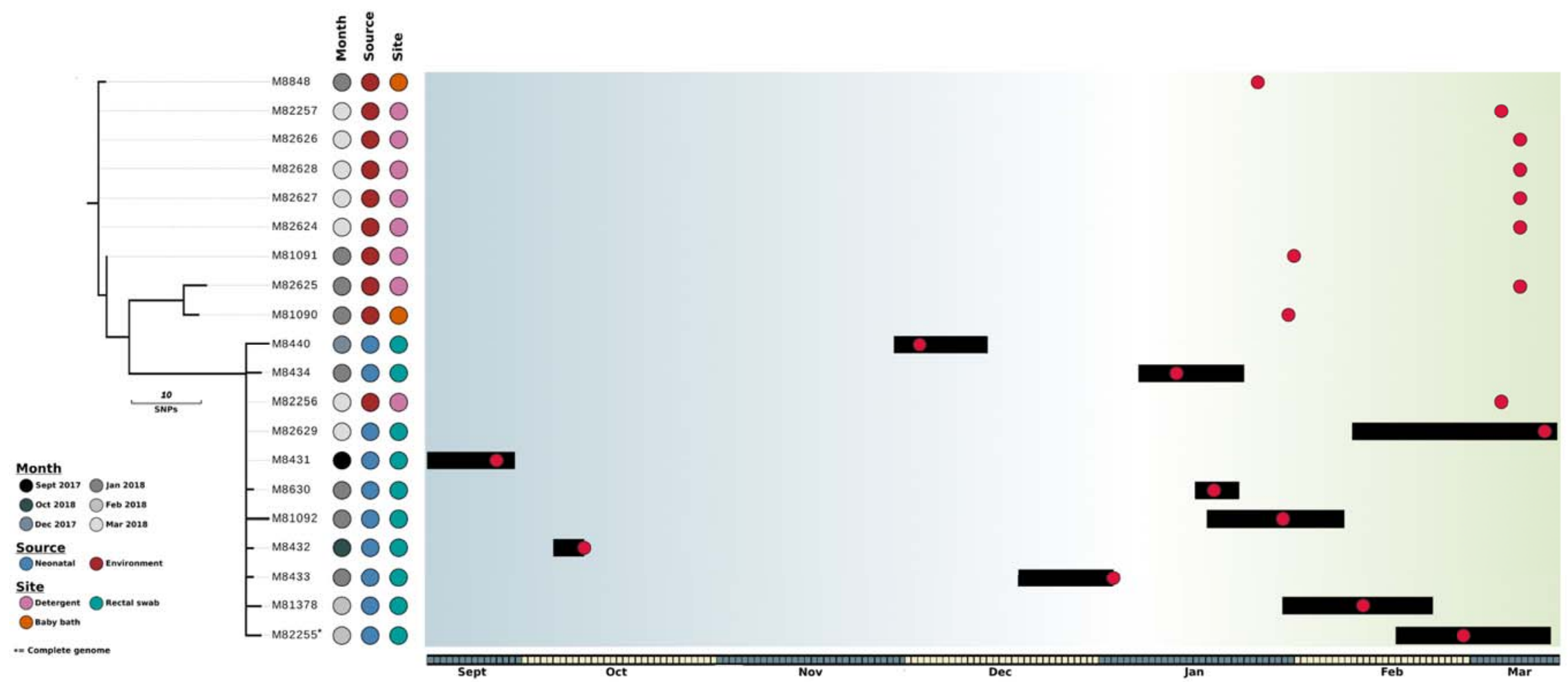

Figure 1: The evolutionary relationship of $\boldsymbol{K}$. michiganensis isolates. Maximum likelihood phylogenetic tree built using 38 core genome

SNPs relative to K. michiganensis strain M82255. The month of isolation, source (neonatal or environmental) and specific site of isolation are indicated as per the legend. Branches (black lines) represent the genetic distance between isolates in terms of SNPs. The scale is indicated.

The outbreak timeline is presented adjacent to the tree. The timescale is represented on the bottom with alternate months colored blue and 
yellow. Each box in the timeline represent a period of 1 day starting at the 15th of September 2017. Back bars represent the admission periods for colonized patients and red dots represent dates on which the samples were isolated (including environmental isolates). Grey shading represent periods where there was no overlap in patient stays and green shading represents periods where multiple colonized patients were present in the SCN at the same time (yellow). 


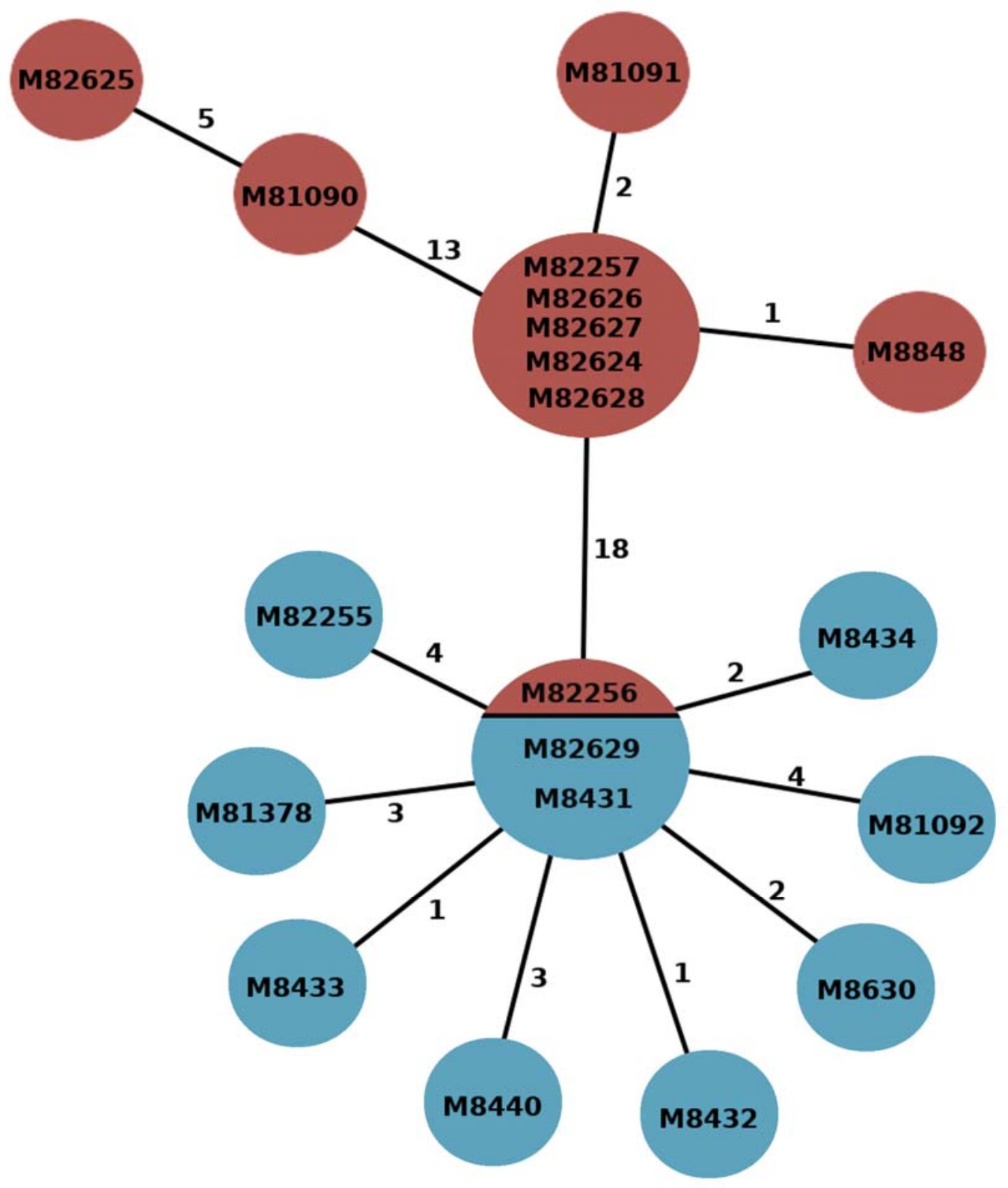

Figure 2: The evolutionary relationship of $K$. michiganensis strains. Minimal Spanning tree showing relationships between environmental (red) and patient (blue) samples. Numbers attached to branches represent number of SNPs between genomes. Isolate codes are represented within each node (if $>1$ code listed, then these were identical at the core genome level). 
Table 1: Sample details

ID

M8431

M8432

M8440

M8433

M8434

M8630

M81092

M81378

M82255

M82629

M8848

M81090

M81091

M82256

M82257

M82624

M82625

M82626

M82627

M82628

\section{SOURCE}

Neonatal

Neonatal

Neonatal

Neonatal

Neonatal

Neonatal

Neonatal

Neonatal

Neonatal

Neonatal

Environmental

Environmental

Environmental

Environmental

Environmental

Environmental

Environmental

Environmental

Environmental

Environmental
GENDER DATE SAMPLE

Male September-2017

Female October-2017

Male

Female

Female

Female

Female

Male

Female

Female

\section{December-2017}

January-2018

January-2018

January-2018

January-2018

February-2018

February-2018

March-2018

January-2018

January-2018

January-2018

March-2018

March-2018

March-2018

March-2018

March-2018

March-2018

March-2018

\section{SAMPLE}

Rectal swab

Rectal swab

Skin swab

Rectal swab

Rectal swab

Rectal swab

Rectal swab

Rectal swab

Rectal swab

Rectal swab

Baby bath drain

Baby Bath

Detergent (Flower service)

Detergent (Milk Room)

Detergent (Nurses Station)

ICU Detergent Brush

Detergent (Pan Room) Milk Room

Detergent (ICU)

Detergent (Pediatric ward)

\section{ORGANISM}

Klebsiella michiganensis

Klebsiella michiganensis

Klebsiella michiganensis

Klebsiella michiganensis

Klebsiella michiganensis

Klebsiella michiganensis

Klebsiella michiganensis

Klebsiella michiganensis

Klebsiella michiganensis

Klebsiella michiganensis

Klebsiella michiganensis

Klebsiella michiganensis

Klebsiella michiganensis

Klebsiella michiganensis

Klebsiella michiganensis

Klebsiella michiganensis

Klebsiella michiganensis

Klebsiella michiganensis

Klebsiella michiganensis

Klebsiella michiganensis
Hospital

Caboolture hospital

Caboolture hospital

External hospital

Caboolture hospital

Caboolture hospital

Caboolture hospital

Caboolture hospital

Caboolture hospital

Caboolture hospital

Caboolture hospital

Caboolture hospital

Caboolture hospital

Caboolture hospital

Caboolture hospital

Caboolture hospital

Caboolture hospital

Caboolture hospital

Caboolture hospital

Caboolture hospital

Caboolture hospital 


\section{References}

1. Stapleton PJ, Murphy M, McCallion N, Brennan M, Cunney R, Drew RJ.

2016. Outbreaks of extended spectrum beta-lactamase-producing

Enterobacteriaceae in neonatal intensive care units: a systematic review. Arch Dis Child Fetal Neonatal Ed 101:F72-78.

2. Saha R, Farrance CE, Verghese B, Hong S, Donofrio RS. 2013. Klebsiella michiganensis sp. nov., a new bacterium isolated from a tooth brush holder. Curr Microbiol 66:72-78.

3. Zheng B, Xu H, Yu X, Lv T, Jiang X, Cheng H, Zhang J, Chen Y, Huang C, Xiao Y. 2018. Identification and genomic characterization of a KPC-2-, NDM1- and NDM-5-producing Klebsiella michiganensis isolate. J Antimicrob Chemother 73:536-538.

4. Hazen TH, Mettus R, McElheny CL, Bowler SL, Nagaraj S, Doi Y, Rasko DA. 2018. Diversity among blaKPC-containing plasmids in Escherichia coli and other bacterial species isolated from the same patients. Sci Rep 8:10291.

5. Founou RC, Founou LL, Allam M, Ismail A, Essack SY. 2018. Genomic characterisation of Klebsiella michiganensis co-producing OXA-181 and NDM-1 carbapenemases isolated from a cancer patient in uMgungundlovu District, KwaZulu-Natal Province, South Africa. S Afr Med J 109:7-8.

6. Seiffert SN, Wuthrich D, Gerth Y, Egli A, Kohler P, Nolte O. 2019. First clinical case of KPC-3-producing Klebsiella michiganensis in Europe. New Microbes New Infect 29:100516.

7. Lowe C, Willey B, O'Shaughnessy A, Lee W, Lum M, Pike K, Larocque C, Dedier H, Dales L, Moore C, McGeer A, Mount Sinai Hospital Infection Control T. 2012. Outbreak of extended-spectrum beta-lactamase-producing Klebsiella oxytoca infections associated with contaminated handwashing sinks. Emerg Infect Dis 18:1242-1247. 
8. Mete B, Aybar Bilir Y, Aygun G, Yilmaz M, Urkmez S, Dilmen Y, Ozturk R. 2013. Klebsiella oxytoca outbreak in an intensive care unit: a probable link to common insulin vial use. Anaesth Intensive Care 41:266-268.

9. Vergara-Lopez S, Dominguez MC, Conejo MC, Pascual A, RodriguezBano J. 2013. Wastewater drainage system as an occult reservoir in a protracted clonal outbreak due to metallo-beta-lactamase-producing Klebsiella oxytoca. Clin Microbiol Infect 19:E490-498.

10. Leitner E, Zarfel G, Luxner J, Herzog K, Pekard-Amenitsch S, Hoenigl M, Valentin T, Feierl G, Grisold AJ, Hogenauer C, Sill H, Krause R, ZollnerSchwetz I. 2015. Contaminated handwashing sinks as the source of a clonal outbreak of KPC-2-producing Klebsiella oxytoca on a hematology ward. Antimicrob Agents Chemother 59:714-716.

11. Schmithausen RM, Sib E, Exner M, Hack S, Rosing C, Ciorba P, Bierbaum G, Savin M, Bloomfield SF, Kaase M, Jacobshagen A, Gemein S, Gebel J, Engelhart S, Exner D. 2019. The Washing Machine as a Reservoir for Transmission of Extended-Spectrum-Beta-Lactamase (CTX-M15)-Producing Klebsiella oxytoca ST201 to Newborns. Appl Environ Microbiol 85:e01435-01419.

12. Arakawa Y, Ohta M, Kido N, Mori M, Ito H, Komatsu T, Fujii Y, Kato N. 1989. Chromosomal beta-lactamase of Klebsiella oxytoca, a new class A enzyme that hydrolyzes broad-spectrum beta-lactam antibiotics. Antimicrobial Agents and Chemotherapy 33:63-70.

13. Fevre C, Jbel M, Passet V, Weill FX, Grimont PA, Brisse S. 2005. Six groups of the OXY beta-Lactamase evolved over millions of years in Klebsiella oxytoca. Antimicrob Agents Chemother 49:3453-3462.

14. Joris B, De Meester F, Galleni M, Frere JM, Van Beeumen J. 1987. The K1 beta-lactamase of Klebsiella pneumoniae. Biochem J 243:561-567. 
15. Fournier B, Lagrange PH, Philippon A. 1996. beta-lactamase gene promoters of 71 clinical strains of Klebsiella oxytoca. Antimicrob Agents Chemother 40:460-463.

16. Jeong SH, Kim WM, Chang CL, Kim JM, Lee K, Chong Y, Hwang HY, Baek YW, Chung HK, Woo IG, Ku JY. 2001. Neonatal intensive care unit outbreak caused by a strain of Klebsiella oxytoca resistant to aztreonam due to overproduction of chromosomal beta-lactamase. J Hosp Infect 48:281-288.

17. Zarate MS, Gales AC, Picao RC, Pujol GS, Lanza A, Smayevsky J. 2008. Outbreak of OXY-2-Producing Klebsiella oxytoca in a renal transplant unit. J Clin Microbiol 46:2099-2101.

18. Then RL, Glauser MP, Angehrn P, Arisawa M. 1983. Cephalosporin resistance in strains of Klebsiella oxytoca isolated during antibiotic therapy. Zentralbl Bakteriol Mikrobiol Hyg A 254:469-479.

19. Potz NA, Colman M, Warner M, Reynolds R, Livermore DM. 2004. Falsepositive extended-spectrum beta-lactamase tests for Klebsiella oxytoca strains hyperproducing K1 beta-lactamase. J Antimicrob Chemother 53:545547.

20. Bush K, Jacoby GA. 2010. Updated functional classification of betalactamases. Antimicrob Agents Chemother 54:969-976.

21. Hart CA. 1993. Klebsiellae and neonates. J Hosp Infect 23:83-86.

22. Zaidi AK, Huskins WC, Thaver D, Bhutta ZA, Abbas Z, Goldmann DA. 2005. Hospital-acquired neonatal infections in developing countries. Lancet $365: 1175-1188$.

23. European Committee on Antimicrobial Susceptibility Testing. 2017. EUCAST guidelines for detection of resistance mechanisms and specific resistances of clinical and/or epidemiological importance, version 2.0. EUCAST. 
24. Bolger AM, Lohse M, Usadel B. 2014. Trimmomatic: a flexible trimmer for Illumina sequence data. Bioinformatics 30:2114-2120.

25. Gupta SK, Padmanabhan BR, Diene SM, Lopez-Rojas R, Kempf M, Landraud L, Rolain JM. 2014. ARG-ANNOT, a new bioinformatic tool to discover antibiotic resistance genes in bacterial genomes. Antimicrob Agents Chemother 58:212-220.

26. Inouye M, Dashnow H, Raven LA, Schultz MB, Pope BJ, Tomita T, Zobel J, Holt KE. 2014. SRST2: Rapid genomic surveillance for public health and hospital microbiology labs. Genome Med 6:90.

27. Croucher NJ, Page AJ, Connor TR, Delaney AJ, Keane JA, Bentley SD, Parkhill J, Harris SR. 2015. Rapid phylogenetic analysis of large samples of recombinant bacterial whole genome sequences using Gubbins. Nucleic Acids Res 43:e15.

28. Wood DE, Salzberg SL. 2014. Kraken: ultrafast metagenomic sequence classification using exact alignments. Genome Biol 15:R46.

29. Federhen S, Rossello-Mora R, Klenk H-P, Tindall BJ, Konstantinidis KT, Whitman WB, Brown D, Labeda D, Ussery D, Garrity GM, Colwell RR, Hasan N, Graf J, Parte A, Yarza P, Goldberg B, Sichtig H, KarschMizrachi I, Clark K, McVeigh R, Pruitt KD, Tatusova T, Falk R, Turner S, Madden T, Kitts P, Kimchi A, Klimke W, Agarwala R, DiCuccio M, Ostell J. 2016. Meeting report: GenBank microbial genomic taxonomy workshop (12-13 May, 2015). Standards in Genomic Sciences 11.

30. Ronning TG, Aas CG, Stoen R, Bergh K, Afset JE, Holte MS, Radtke A. 2019. Investigation of an outbreak caused by antibiotic-susceptible Klebsiella oxytoca in a neonatal intensive care unit in Norway. Acta Paediatr 108:76-82.

31. Ransjo U, Good Z, Jalakas K, Kuhn I, Siggelkow I, Aberg B, Anjou E. 1992. An outbreak of Klebsiella oxytoca septicemias associated with the use 
of invasive blood pressure monitoring equipment. Acta Anaesthesiol Scand 36:289-291.

32. Toldos CM, Ortiz G, Camara M, Segovia M. 1997. Application of pulsedfield gel electrophoresis in an outbreak of infection due to Klebsiella oxytoca. J Med Microbiol 46:889-890.

33. Watson JT, Jones RC, Siston AM, Fernandez JR, Martin K, Beck E, Sokalski S, Jensen BJ, Arduino MJ, Srinivasan A, Gerber SI. 2005. Outbreak of catheter-associated Klebsiella oxytoca and Enterobacter cloacae bloodstream infections in an oncology chemotherapy center. Arch Intern Med 165:2639-2643.

34. Tindall BJ, Rossello-Mora R, Busse HJ, Ludwig W, Kampfer P. 2010. Notes on the characterization of prokaryote strains for taxonomic purposes. Int J Syst Evol Microbiol 60:249-266.

35. Liao TL, Lin AC, Chen E, Huang TW, Liu YM, Chang YH, Lai JF, Lauderdale TL, Wang JT, Chang SC, Tsai SF, Chen YT. 2012. Complete genome sequence of Klebsiella oxytoca E718, a New Delhi metallo-betalactamase-1-producing nosocomial strain. J Bacteriol 194:5454.

36. Reiss I, Borkhardt A, Füssle R, Sziegoleit A, Gortner L. 2000. Disinfectant contaminated with Klebsiella oxytoca as a source of sepsis in babies. The Lancet 356:310.

37. Paczosa MK, Mecsas J. 2016. Klebsiella pneumoniae: Going on the Offense with a Strong Defense. Microbiol Mol Biol Rev 80:629-661.

38. Shukor MY, Husin WS, Rahman MF, Shamaan NA, Syed MA. 2009. Isolation and characterization of an SDS-degrading Klebsiella oxytoca. J Environ Biol 30:129-134. 